\section{CRISPR therapies - making the grade not the cut}

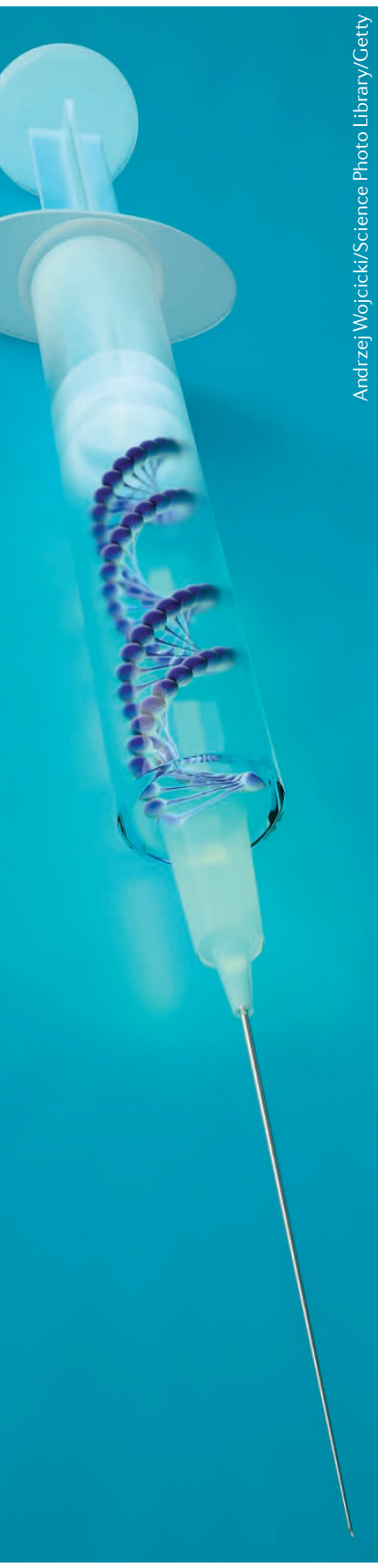

CRISPR-based systems for therapeutic purposes have shown promise in numerous mouse models of human diseases, but one concern that is limiting roll-out to human trials is that during genome editing, unintended Cas9-induced DNA double-strand breaks (DSBs) could cause off-target mutations, toxicity and harm to patients. A new study adapts CRISPRbased transcriptional modulation as a potential DSB-free system with in vivo therapeutic efficacy in various mouse models of human diseases.

Modulation of transcription by CRISPR systems typically involves a nuclease-dead Cas9 (dCas9) fused to a protein domain that alters transcription, such as a transcriptional activator, repressor or histone modifier. This fusion protein is then guided to a target gene by a designed single-guide RNA (sgRNA). Liao et al. sought to overcome a major technical challenge in making this system clinically deliverable: the most suitable type of non-integrating gene therapy vector, adeno-associated virus (AAV), has limited packaging capacity and so cannot accommodate genes encoding typical dCas9 fusion proteins. Instead, the authors adapted the sgRNA, primarily by including MS2 loops as an independent means of recruiting the engineered transcriptional activator protein MS2-P65-HSF1 (MPH) without requiring fusion to dCas9. Furthermore, the sgRNA was truncated so that even when bound by wild-type Cas9 the complex is essentially nuclease-dead at a genomic target site; the authors termed these dead sgRNAs (dgRNAs). This system allows the CRISPR-based transcriptional activation components to be feasibly encoded in separate AAVs: one for both the dgRNA and the $\mathrm{MPH}$ transcriptional activator and one for Cas9.

The team then tested whether their system could ameliorate symptoms in various mouse models of disease; in most cases, their strategy was to use cell culture systems to identify dgRNAs conferring potent target-gene activation and then use single AAV vectors to co-deliver the dgRNA and MPH (either systemically via the bloodstream or through local injection into specific organs) to mice already expressing wild-type Cas 9 transgenically.

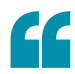

This system allows the ... components to be feasibly encoded in separate AAVs

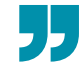

They demonstrated that upregulation of interleukin-10 (Il10) or klotho $(\mathrm{Kl})$ ameliorated the effects of subsequent cisplatin-induced kidney injury; that upregulation of pancreatic and duodenal homeobox gene $1(P d x 1)$ in the liver caused insulin production by liver cells and partial rescue of streptozotocin-induced hyperglycaemia (a mouse model of type 1 diabetes mellitus); and that upregulation of utrophin (Utrn) (either neonatally or after disease onset) reduced muscle weakness in the $m d x$ genetic model of Duchenne muscular dystrophy, which is caused by a dysfunctional dystrophin gene. The ability to overexpress large genes such as utrophin is noteworthy, as the size of the cDNA, which most vectors cannot accommodate, makes this difficult to achieve by standard gene therapy approaches.

Importantly, adoption into human clinical care would require co-delivery of dgRNAs, MPH and Cas9. Following a demonstration that upregulating follistatin $(F s t)$ increases muscle mass in Cas9-expressing mice, the authors showed that muscle co-injection of AAVs encoding dgFst-MPH and Cas9, or dgUtrn-MPH and Cas9, ameliorated disease symptoms in $m d x$ mice that did not already express Cas9.

Going forwards, it will be interesting to see whether wild-type Cas9 or dCas9 will be the most suitable Cas9 variant. In their study, Liao et al. showed that, when tested in the same experiment, wild-type Cas9 and dCas 9 conferred equivalent transcriptional activation of Fst. They also did not detect DSBs induced by wild-type Cas 9 using a Surveyor assay coupled to deep sequencing; however, dCas9 might be the favoured variant in future as an added safeguard against DSBs, provided that it demonstrates continued transcriptional equivalency to wild-type Cas9 in wider tests.

Overall, this system has numerous attractive features as a potential human therapeutic for various disorders, particularly the lack of overt genome modification by either Cas9 or the non-integrating AAV vector. However, with such properties it will be important to determine whether therapeutic benefit can be achieved long term, such as how long transcriptional modulation can be sustained after a single injection, and whether the potency of serial injections will diminish through immune responses to AAV or its payloads.

Darren J. Burgess, Senior Editor, Nature Reviews Genetics This article is modified from the original in Nat. Rev. Genetics (http://dx.doi.org/10.1038/nrg.2017.112) 